\title{
Brugada syndrome and carbohydrate metabolism
}

\begin{abstract}
Brugada syndrome is an arrhythmogenic and autosomal dominant disease with incomplete penetrance that may cause syncope and sudden cardiac death in young individuals with a normal heart. In addition, it is the syndrome of right bundle branch block (RBBB) that characterized by ST-segment elevation in precordial ECG leads V1 to V3. There are many factors contributing to the syndrome, including genetic factors, cellular and ionic mechanisms abnormalities, exercise, carbohydrate (CHO) and blood electrolyte. Thai ministry of Public Health reported in 1990 that there was an association between a large meal of glutinous ("sticky") rice or carbohydrates ingested on the night of death in Brugada syndrome victims. Some investigators have reported that a glucose load, glucose alone, glucose combined with insulin infusion, and Thai high glycemic index (HGI) meals influenced ST-segment elevation in Brugada syndrome patients. A reduction in INa or ICaL or an increase in Ito and/or any other potassium current may cause the changes of the configuration of the action potential notch that leads to J-point elevation on the ECG. Food ingestion is accompanied by the release of hormones such as insulin, glucagon and gut peptides. A larger meal produced a longer gastric emptying and a higher effect on postprandial profile of the gut hormone. Insulin is an essential hormone that controls the level of BG. Food ingestion activated sympathetic activity indicating by an increased postprandial plasma noradrenaline. increased postprandial sympathetic nerve activity muscle. In addition, it was known that HGI and carbohydrate load contribute to high $\mathrm{CHO}$ oxidation rate. According to the above information, the increasing of digestive related hormones and the $\mathrm{CHO}$ oxidation rate induced by having HGI or $\mathrm{CHO}$ load may result in abnormal metabolic and autonomic responses in these patients.
\end{abstract}

Keywords: brugada syndrome, electrocardiogram, electrolyte, heart rate variability, glycemic index
Volume 8 Issue 5 - 2017

\author{
Raoyring Chanavirut,' Naruemon \\ Leelayuwat ${ }^{2,3}$ \\ 'Physical Therapy Division, Faculty of Associated Medical \\ Sciences, Khon Kaen University, Thailand \\ ${ }^{2}$ Department of Medicine, Faculty of Medicine, Khon Kaen \\ University, Thailand \\ ${ }^{3}$ Exercise and Sport Sciences Development and Research Group, \\ Khon Kaen University, Thailand
}

Correspondence: Naruemon Leelayuwat, Department of Physiology, Faculty of Medicine, Khon Kaen University, Khon Kaen, 40002, Thailand, Tel +66 43348 394, Fax +66 43348 394, Email naruemon@kku.ac.th

Received: March 31, 2017 | Published: April 18, 2017
Abbreviations: SUDS, sudden unexpected death syndrome; ANS, autonomic nervous system; CHO, carbohydrate; HGI, high glycaemic index; ECG, electrocardiography; SCD, sudden cardiac death; RBBB, right bundle branch block; GI, glycaemic index; BG, blood glucose; OGTT, oral glucose tolerance test

\section{Introduction}

\section{Brugada syndrome}

Brugada syndrome is recognized worldwide, although the prevalence seems much higher in some areas. In Asia, the plenty of cases were reported from Japan, Thailand, Taiwan, China, India, Laos, Vietnam, Singapore and Cambodia. The incidence of the syndrome was nine times higher among men than women and the average age was $45 \pm 10.5$ years. ${ }^{1}$ For Southeast Asian countries, Brugada syndrome was the major cause of sudden death in younger population. ${ }^{2,3}$ In Thailand, an epidemiological survey of young men living along the northeastern border of Thailand abutting Laos and Cambodia found that the annual SUDS death rate was 26-38 per 100,000 men (range, 20-49years old). ${ }^{4,5}$

Brugada syndrome is an arrhythmogenic and autosomal dominant disease with incomplete penetrance that may cause syncope and sudden cardiac death (SCD) in young individuals with a normal heart. ${ }^{6}$ In addition, it is the syndrome of right bundle branch block (RBBB) that characterized by ST-segment elevation in precordial ECG leads V1 to V3. ${ }^{7}$ There are many factors contributing to the syndrome, including genetic factors, cellular and ionic mechanisms abnormalities, gender difference, exercise, autonomic nervous system, and blood electrolyte. ${ }^{8}$ A genetic mechanism explaining the pathology of the syndrome is beyond this review.

\section{Brugada syndrome and carbohydrate metabolism}

Based on Thai ministry of Public Health report in 1990 there was an association between a large meal of glutinous ("sticky") rice or carbohydrates ingested on the night of death in Brugada syndrome victims. ${ }^{9}$ This report motivated many investigators to approve that association. In general, glutinous rice (Oryza sativa) is one type of carbohydrate staple food of Asian people. Glycaemic index (GI) value is defined as blood glucose produced after the ingestion of a carbohydrate food..$^{10}$ In fact, there are large ranges of GI of rice which aredue to variations of rice's species, ${ }^{11}$ process conditions ${ }^{12,13}$ and differences in amylose and amylopectin content. The GI value of the glutinous rice when compared with glucose is $98^{14}$ and $\mathrm{CHO}$ composition per $100 \mathrm{~g}$ of the glutinous rice is $52 \mathrm{~g} .{ }^{15}$

Some investigators have reported that a glucose load, ${ }^{16}$ glucose alone,${ }^{16}$ glucose combined with insulin infusion,${ }^{17}$ and Thai HGI meals ${ }^{18}$ influenced ST-segment elevation in Brugada syndrome patients. It has been known that the epicardial action potential notch is the result of the balance between three different currents: INa, Ito and ICaL. ${ }^{19}$ Theoretically, a reduction in INa or ICaL or an increase in Ito and/or any other potassium current may cause the changes of the configuration of the action potential notch ${ }^{19,20}$ that leads to J-point elevation on the ECG. In the general concept, glucose induces insulin secretion. From many previous studies, insulin has been shown to cause hyperpolarization of membrane potential by activation of the $\mathrm{Na}+$ and $\mathrm{K}+$ pump, ${ }^{20,21}$ which activated outward current during the plateau phase of action potential. Therefore, it tends to promote outward current. In addition, activation of the $\mathrm{Na}+$ and $\mathrm{K}+$ pump also could produce a high $\mathrm{K}+$ gradient across the cell membrane with depletion of $\mathrm{K}+$ from the extracellular space. However, the study of Nishizaki and co-workers found serum $\mathrm{K}+$ concentrations were unchanged after 
the glucose load. They still proposed the possibility that increased $\mathrm{K}+$ gradient could affect the repolarization process differently among the three regions of the ventricular walls (endocardium, epicardium and myocardium) cannot be excluded and might contribute to the STsegment abnormalities observed in patients with Brugada syndrome. ${ }^{16}$

Aulbach and co-workers have reported that insulin stimulates the ICaL in isolated rat ventricular myocytes in a dose-dependent manner. ${ }^{22}$ Therefore, it tends to promote inward current that thought to reduce ST-segment elevation. ${ }^{23,24}$ Until now, it seems to be not fully understood how insulin affects the repolarizing currents. However, Nishizaki and co-workers suggested that an overall insulin effect on ST-segment elevation in Brugada syndrome patients might be a balance between the modulation of $\mathrm{Na}+$ and $\mathrm{K}+$ pump current and $\mathrm{ICaL}$ and additional influences on other channels or regional differences of its effects on repolarizing currents. Moreover, the report from previous studies proposed that the glucose load would change the characteristics of other factors, such as the ANS, plasma viscosity, and other ion channels ${ }^{25}$ which might influence ST segment to fluctuate occasionally in Brugada syndrome patients. Thereafter, it needed to be explored further.

Due to the Brugada ECG which was characterized by a prominent coved ST-segment elevation displaying J wave amplitude or STsegment elevation $\geq 2 \mathrm{~mm}$ or $0.2 \mathrm{mV}$ at its peak followed by a negative T-wave, with little or no isoelectric separation the earlier study by Nogami and co-workers investigated infusion of glucose or glucose and insulin solution then identified the abnormality of ECG. They found the accentuation of ST-segment in high-risk Brugada syndrome patients. However, the changes of blood glucose (BG) in this study were not directly related to the ECG changes. ${ }^{17}$ Another study by Nishizaki assessed oral glucose tolerance test (OGTT) in Brugada syndrome patients and control healthy subjects. They found Glucose-induced insulin secretion lead to ST-segment elevation and induce ECG morphologic changes only in patients whereas BG, insulin level and $\mathrm{K}+$ level were not significantly different between two groups. ${ }^{16}$ Ikeda et al., ${ }^{26}$ demonstrated that the augmentation of ST-segment elevation was associated with a stomach rapidly filled with a large meal. ${ }^{26}$ Furthermore, another study indicated that, for symptomatic Brugada syndrome patients, bradycardia-dependent augmentation of ST-segment elevation characteristic was enhanced during the postprandial period, especially after dinner. ${ }^{27}$ In addition, other investigator group from Thailand investigated the effect of the ingestion of three Thai regular diets on day 1 and three HGI diet on day 2 on the maximum ST-segment elevation after each meal in Brugada syndrome patients. The results from this study indicated that HGI meals lead to ST-segment elevation at immediate and at $60 \mathrm{~min}$ after the meal. Moreover, the difference was found only in male participants. However, the insulin and BG level were not reported. ${ }^{18}$ Therefore, both blood parameters according to the GI meals should be further explored.

As is widely known, food ingestion is accompanied by the release of the wide range of hormones such as insulin, glucagon and gut peptides, in which their primary role is controlling digestive function. A larger meal produced a longer gastric emptying and a higher effect on postprandial profile of the gut hormone. ${ }^{28}$ Insulin is an essential hormone that controls the level of BG. It stimulated sympathetic activity both in central and peripheral levels. ${ }^{29}$ Based on the finding from previous studies, food ingestion activated sympathetic activity indicating by an increased postprandial plasma noradrenaline, ${ }^{30}$ increased postprandial sympathetic nerve activity muscle. ${ }^{31}$ In addition, it was known that $\mathrm{HGI}$ and $\mathrm{CHO}$ load contribute to high $\mathrm{CHO}$ oxidation rate. ${ }^{32}$ According to the above information, an increasing of digestive related hormones and the $\mathrm{CHO}$ oxidation rate induced by having HGI or CHO load may result in abnormal metabolic and autonomic responses in these Brugada Syndrome patients.

\section{Conclusion}

This review shows that there are many factors contributing to the syndrome, including genetic factors, cellular and ionic mechanisms abnormalities, gender difference, exercise, autonomic nervous system, and blood electrolyte. ${ }^{8}$ In addition, an increasing of digestive related hormones and the $\mathrm{CHO}$ oxidation rate induced by having HGI or $\mathrm{CHO}$ load may result in abnormal metabolic and autonomic responses in these Brugada Syndrome patients.

\section{Acknowledgments}

None.

\section{Conflicts of interest}

Author declares there are no conflicts of interest.

\section{Funding}

None.

\section{References}

1. Matsuo K,Akahoshi M, Nakashima E, et al. The prevalence, incidence and prognostic value of the Brugada-type electrocardiogram. A populationbased study of four decades. J Am Coll Cardiol. 2001;38(2):765-770.

2. Nademanee K. Sudden unexplained death syndrome in Southeast Asia. Am J Cardiol. 1997;(79):10-11.

3. Vatta M, Dumaine R, Varghese G, et al. Genetic and biophysical basis of sudden unexplained nocturnal death syndrome (SUNDS), a disease allelic to Brugada syndrome. Hum Mol Gene. 2002;11(3):337-345.

4. Tungsanga K, Sriboonlue P. Sudden unexplained death syndrome in north-east Thailand. Int J Epidemio. 1993;22(1):81-87.

5. Tatsanavivat P, Chirawatkul A, Klungboonkrong V, et al. Sudden and unexplained deaths in sleep (Lai Tai) of young men in rural northeastern Thailand. Int J Epidemio. 1992;21(5):904-910.

6. Francis J, Antzelevitch C. Brugada syndrome. Int J Cardiol 2005; 101:173-178.

7. Brugada P, Brugada J. Right bundle branch block, persistent ST segment elevation and sudden cardiac death: a distinct clinical and electrocardiographic syndrome. A multicenter report. J Am Coll Cardiol. 1992;20(6):1391-1396.

8. Antzelevitch C, Brugada P, Borggrefe M, et al. Brugada syndrome. Report of the second consensus conference. Endorsed by the Heart Rhythm Society and the European Heart Rhythm Association. Circulation. 2005;111(5):659-670.

9. Nimmannit S, Malasit P, Chaovakul V, et al. Pathogenesis of sudden unexplained nocturnal death (lai tai) and endemic distal renal tubular acidosis. Lancet. 1991;338(8772):930-932.

10. Jenkins DJ, Wolever TM, Taylor RH, et al. Glycemic index of foods: a physiological basis for carbohydrate exchange. Am J Clin Nutr. 1981;34(3):362-366.

11. Miller JB, Pang E, Bramall L. Rice: a high or low glycemic index food? Am J Clin Nutr. 1992;56(6):1034-1036.

12. Brand JC, Nicholson PL, Thorburn AW, et al. Food processing and the glycemic index. Am J Clin Nutr. 1985;(42):1192-1196. 
13. Traianedes K, O’Dea K. Commercial canning increases the digestibility of beans in vitro and postprandial metabolic responses to them in vivo. Am J Clin Nutr. 1986;(44):390-397.

14. Foster-Powell K, Holt SH, Brand-Miller JC. International table of glycemic index and glycemic load values. Am J Clin Nutr. 2002;76:5-56.

15. Puwastien P. Food composition database for Inmucal program. Institute of nutrition, Mahidol University. 2005.

16. Nishizaki M, Sakurada H, Ashikaga T, et al. Effects of glucose-induced insulin secretion on ST segment elevation in the Brugada syndrome. $J$ Cardiovasc Electrophysiol. 2003;14(3):243-249.

17. Nogami A, Nakao M, Kubota S, et al. Enhancement of J-ST-segment elevation by the glucose and insulin test in Brugada syndrome. Pacing Clin Electrophysiol . 2003;26(1 Pt 2):332-337.

18. Makarawate P, Sangwatanaroj S. Effect of high glycemic index diets on ST segment elevation in Brugada syndrome. Asean Heart Journal. 2008; 16:57-62.

19. Antzelevitch C. The Brugada syndrome. J Cardiovasc Electrophysiol . 2006;9(5):513-516

20. Sassone B, Sacca` S, Mario Donateo. Paradoxical effect of ajmaline in a patient with Brugada syndrome. Europace 8: 251-254.

21. LaManna VR, Ferrier GR (1981) Electrophysiological effects of insulin on normal and depressed cardiac tissues. Am J Physiol. 2006;240(4):H636-H644.

22. Aulbach F, A Simm, Maier S, Langenfeld H, et al. Insulin Stimulates the L-Type Ca2+ Current in Rat Cardiac Myocytes. Cardiovasc Res. 1999;42(1):113-120.
23. Antzelevitch C. The Brugada Syndrome: Ionic Basis and Arrhythmia Mechanisms. J Cardiovascular Electrophysiology. 2001;12(2):268-272.

24. Yan GX, Antzelevitch C. Cellular basis for the electrocardiographic J wave. Circulation. 1996;93(2):372-379.

25. Rowe JW, Young JB, Minaker KL, et al. Effect of insulin and glucose infusions on sympathetic nervous system activity in normal man. Diabetes. 1981;30(3):219-225.

26. Ikeda T, Abe A, Yusu S, et al. The full stomach test as a novel diagnostic technique for identifying patients at risk of Brugada syndrome. $J$ Cardiovasc Electrophysiol. 2006;17(6):602-607.

27. MizumakiI K, Fujiki A, Nishida K, et al. Postprandial Augmentation of Bradycardia-Dependent ST Elevation in Patients with Brugada Syndrome. J Cardiovasc Electrophysiol. 2007;18(8):834-844.

28. Sidery MB, Macdonald IA. The effect of meal size on the cardiovascular responses to food ingestion. Br J Nutr. 1994;71(6):835-848.

29. Porte D, Baskin GD, Schwartz MW. Insulin signaling in the central nervous system: a critical role in metabolic homeostasis and disease from C. elegans to humans. Diabetes. 2005;54(5):1264-1276.

30. Heseltine D, Potter JF, Hartley G, et al. Blood pressure, heart rate and neuroendocrine responses to a high carbohydrate and a high fat meal in healthy young subjects. Clin Sci (Lond) . 1991;79(5):517-522.

31. Fagius $\mathrm{J}$ and Berne $\mathrm{C}$. Increase in muscle nerve sympathe tic activity in humans after food intake. Clin Sci. 1994;86(2):159-167.

32. Wu CL, Nicholas C, Williams C, et al. The influence of high-carbohydrate meals with different glycaemic indices on substrate utilisation during subsequent exercise. Br J Nutr. 2003; 90(6):1049-1056. 